\title{
Model-Based Control of an Aircraft Engine using an Optimal Tuner Approach
}

\author{
Joseph W. Connolly* \\ NASA Glenn Research Center, \\ Cleveland, $\mathrm{OH}$ 44135, USA
}

\author{
Amy Chicatelli ${ }^{\dagger}$ \\ Vantage Partners LLC, \\ Brook Park, OH 44142, USA
}

\author{
Sanjay Garg \\ NASA Glenn Research Center, Cleveland, OH 44135, USA
}

\begin{abstract}
This paper covers the development of a model-based engine control (MBEC) methodology applied to an aircraft turbofan engine. Here, a linear model extracted from the Commercial Modular Aero-Propulsion System Simulation 40,000 (CMAPSS40k) at a cruise operating point serves as the engine and the on-board model. The on-board model is updated using an optimal tuner Kalman Filter (OTKF) estimation routine, which enables the on-board model to self-tune to account for engine performance variations. The focus here is on developing a methodology for MBEC with direct control of estimated parameters of interest such as thrust and stall margins. MBEC provides the ability for a tighter control bound of thrust over the entire life cycle of the engine that is not achievable using traditional control feedback, which uses engine pressure ratio or fan speed. CMAPSS40k is capable of modeling realistic engine performance, allowing for a verification of the MBEC tighter thrust control. In addition, investigations of using the MBEC to provide a surge limit for the controller limit logic are presented that could provide benefits over a simple acceleration schedule that is currently used in engine control architectures.
\end{abstract}

\section{Nomenclature}

\begin{tabular}{|c|c|c|c|}
\hline$A$ & System matrix & $N_{c}$ & Core rotational speed \\
\hline$B$ & System matrix & $N_{f}$ & Fan rotational speed \\
\hline$C$ & System matrix & OTKF & Optimal tuner Kalman Filter \\
\hline CMAPSS40k & $\begin{array}{l}\text { Commercial modular aero-propulsion } \\
\text { system simulation } 40,000\end{array}$ & $P$ & $\begin{array}{l}\text { Covariance matrix of } \\
\text { estimated parameters }\end{array}$ \\
\hline$D$ & System matrix & P2 & Inlet pressure \\
\hline EPP & Efficient propulsion and power & P5 & Turbine exit pressure \\
\hline EPR & Engine pressure ratio & PI & Proportional integral controller \\
\hline$F$ & System matrix & PLA & Power lever angle \\
\hline$F_{n e t}$ & Engine net thrust & Ps3 & Compressor discharge pressure \\
\hline G & System matrix & $Q$ & Process noise covariance matrix \\
\hline $\mathrm{HPC}$ & High pressure compressor & $R$ & Measurement noise covariance matrix \\
\hline IVHM & Integrated vehicle health management & SFW & Subsonic fixed wing \\
\hline$K$ & Kalman Filter gain & SM & Stall margin \\
\hline$L$ & System matrix & $\mathrm{T} 2$ & Inlet temperature \\
\hline MBEC & Model based engine control & T3 & Compressor discharge temperature \\
\hline$N$ & System matrix & T48 & Turbine exit temperature \\
\hline
\end{tabular}

*Aerospace Engineer, Controls and Dynamics Branch, 21000 Brookpark Rd., 77-1, AIAA Senior Member

$\dagger$ Aerospace Engineer, Controls and Dynamics Branch, 3000 Aerospace Parkway VPL-3, AIAA Senior Member

${ }^{\ddagger}$ Branch Chief, Controls and Dynamics Branch, 21000 Brookpark Rd., 77-1, AIAA Associate Fellow 


$\begin{array}{llll}V^{*} & \begin{array}{l}\text { Transformation matrix relating } \\ \text { health and tuning parameters }\end{array} & y & \begin{array}{l}\text { Measured outputs vector } \\ \text { Estimated outputs vector }\end{array} \\ \text { VBV } & \text { Variable bleed valve } & & \\ \text { VSV } & \text { Variable stator vane } & \text { Subscript } & \\ W_{f} & \text { Fuel flow } & c & \text { Control parameter } \\ e & \text { Error } & x h & \text { Augmented state vector }(x \text { and } h) \\ h & \text { Health parameter vector } & x q & \text { Reduced order State vector }(x \text { and } q) \\ q & \text { Kalman Filter tuning parameter vector } & \\ r & \text { Control setpoint } & \text { Superscripts } & \\ u & \text { Actuator command vector } & \dagger & \text { Estimated value } \\ v & \text { Measurement noise vector } & & \\ w & \text { Process noise vector } & & \end{array}$

\section{Introduction}

$\mathrm{T}_{\mathrm{s}}^{\mathrm{H}}$

HE Efficient Propulsion and Power (EPP) element of the NASA Fundamental Aeronautics Program, Subsonic Fixed Wing (SFW) Project aims to develop technologies to improve the fuel efficiency of commercial aircraft. Conservation of fuel in transportation systems has been identified as a national goal to reduce harmful emissions and is recognized as a responsibility that requires government involvement in order to be achieved. NASA has documented that reduction in air pollution and operational costs can be accomplished by creating technologies that reduce the following: drag, engine-specific fuel consumption and aircraft weight. ${ }^{1}$ Model-based engine control (MBEC), one of the tasks under the SFW-EPP element, is being developed as one of the advanced engine control system methodologies to improve turbofan engine performance and efficiency.

In current engine control architectures, the typical design approach is to regulate a measurable variable such as fan shaft speed $\left(N_{f}\right)$ or engine pressure ratio (EPR), which is strongly correlated with thrust. ${ }^{2}$ This is done since engine thrust is not a measurable parameter from typical on-board engine sensors. Additionally, limits on allowable fuel flow are imposed based on a conservative approach to maintain adequate stall margin and temperature margin for safe operation of the engine throughout its life. This approach results in less efficient engine operation and leads to variations in the engine throttle to thrust response with engine deterioration. As an engine deteriorates with usage, the same throttle setting results in slightly different thrust because the relationship of the regulated variable (Nf or EPR) to thrust changes with engine aging.

In order to keep pace with the increasing demands on engine performance, the control system must operate in a manner that exceeds the capabilities of current control architectures. In the engine research community, MBEC is widely recognized as a means to provide the necessary improved efficiency to reduce engine-specific fuel consumption. ${ }^{3}$ MBEC will enable operation of an engine using a direct feedback of thrust providing the ability to have a tighter control of the main parameter of interest for engine performance at various deterioration levels throughout its life cycle or during unexpected disturbances. In addition, MBEC will allow operation with less conservative safety margins, since all safety margins currently are designed to an end of life engine, whereas an on-board model can provide a more accurate margin for the actual condition of the engine. The reduction in the safety margins for newer engines will increase fuel efficiency, which links to critical NASA aeronautics research goals. Direct control of thrust will help maintain a tight bound on variations in the engine throttle to thrust response as the engine ages. This will potentially increase the engine operating life. In addition, by using an engine model that adapts to changing conditions as they occur, the control system can operate more efficiently. These changing conditions can be attributed to the variations in the flight profile or expected wear and deterioration that the engine will experience during its lifetime of use. MBEC could also be used to accommodate minor faults in the engine if implemented with an on-board diagnostic system. The longer-term pay-off of this research is to have a personalized control for each specific engine, which adapts to the actual condition of the engine to not only maintain more efficient operation throughout its lifetime but also increase its useful operating life.

In this paper, the focus is on the development of a linear MBEC about a cruise operating condition. The MBEC model is comprised of three main components; first an engine or "truth" model, second an on-board estimation of desired unmeasured parameters, and last a controller with limit logic. For the work presented, a linear point model extracted from the Commercial Modular Aero-Propulsion System Simulation 
40,000 (CMAPSS40k) ${ }^{4}$ at a cruise operating point will be the engine model, an optimal tuner Kalman Filter (OTKF) will be used to obtain an estimate of thrust and stall margin, and finally a thrust controller with stall margin protection will be developed. The following sections of this paper will discuss the current engine control architecture, methodologies of the MBEC modeling approach, results for a test case of the closed loop thrust control with surge margin limit logic, comparisons of EPR control to thrust based control, future steps, and conclusions.

\section{Current Control Architecture}

A typical control architecture for a turbofan engine equipped with a full authority digital engine control is shown in Fig. 1. The main interface between the pilot and the control system is the power lever angle (PLA), which is used by the controller to set a required $N_{f}$ or EPR command. Traditional control design is restricted to these parameters due to the sensors and actuators that are implemented in practice. The parameters of interest are not directly measured due to an inability to have on-board thrust or stall margin sensors. The measured $N_{f}$ or EPR is subtracted from the command set point to create an error signal that goes to the controller to determine a required fuel flow $\left(W_{f}\right)$. For the CMAPSS40k simulation the controller is designed as a proportional integral control with integrator windup protection. ${ }^{5}$

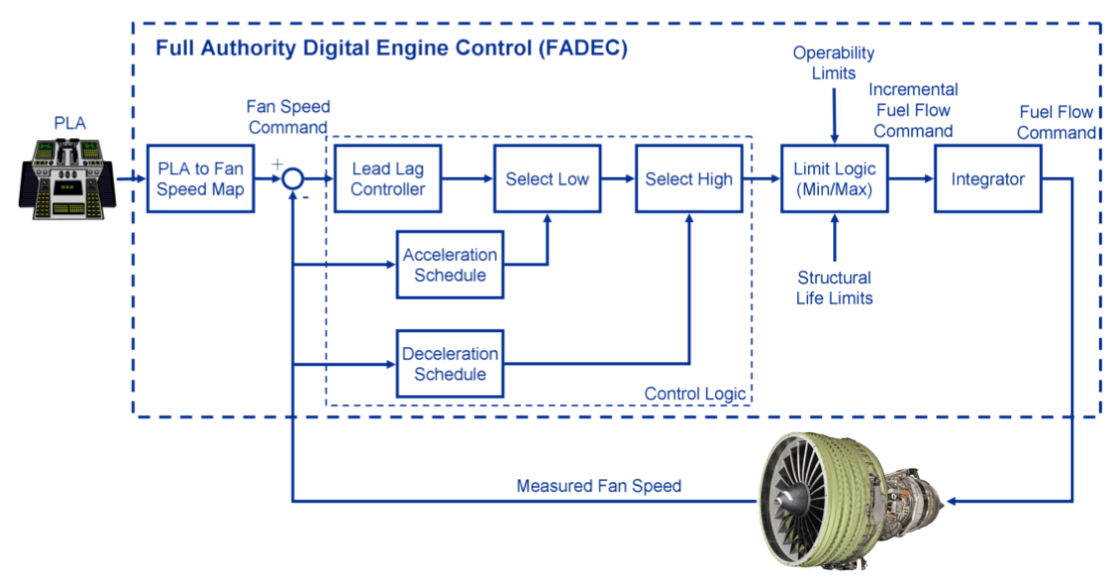

Figure 1. Typical current engine control diagram.
For safety, limit logic is used to regulate excessive temperatures and operating conditions that could lead to stall or other engine failures. This is done by monitoring sensed parameters for engine shafts' acceleration, combustor pressure, maximum shaft speeds, and the ratio of the $W_{f}$ to compressor discharge static pressure. There is a selection process using a $\min / \max$ approach to determine the final $W_{f}$ signal that is provided to the fuel flow injector. While all of these limiters are used in CMAPSS40k, the primary interest in this
the paper will be focused on study is high pressure compressor (HPC) stall, and therefore the rest of the paper will be focused on the rotational acceleration of engine core speed. CMAPSS40k, in addition to controlling the fuel flow, also schedules the variable stator vane (VSV), and variable bleed valve (VBV). Since the VSV and VBV are on open-loop schedules based on the operating condition, they will be assumed to be fixed for the linear model analysis that follows.

\section{Modeling Approach Overview}

The MBEC system architecture will contain an engine simulation, an on-board model with an associated tracking filter for estimating unmeasured parameters, and a controller with limit logic. To accomplish the objective of developing a linear MBEC simulation, the CMAPSS40k cruise linear point model is used as the turbofan engine and the on-board self-tuning model. Within the on-board self-tuning engine model, an OTKF estimation routine is used to tune the on-board model to the current engine condition. The OTKF is based on work performed by Simon, ${ }^{6}$ including its follow-on algorithm development. A general schematic of the MBEC model design is shown in Fig. 2. The figure illustrates a simplified EPR control with core speed acceleration limit logic architecture as discussed in the previous section with the ability to switch to the MBEC design using a thrust controller with stall margin (SM) limit logic. The control parameters for the current control architecture come from the sensors of the CMAPSS40k engine model. To transition from the current control architecture to an MBEC design the control parameters come from the OTKF estimation. This architecture illustrates the current layout of the MBEC model, but also could be used in 
an implementation if it is determined that a traditional control architecture is required as a backup while MBEC is being flight tested.

Although MBEC research has been ongoing for many years by organizations such as General Electric and Pratt and Whitney, ${ }^{7}$ one of the stumbling blocks has been the accuracy of the onboard model as the engine degrades with usage. Typically, engine performance degradation is captured in engine models through health parameters, which are variables such as efficiency and flow capacity that represent performance deterioration within each major rotating component of the engine. The challenge comes about because typically the number of sensors available to update the on-

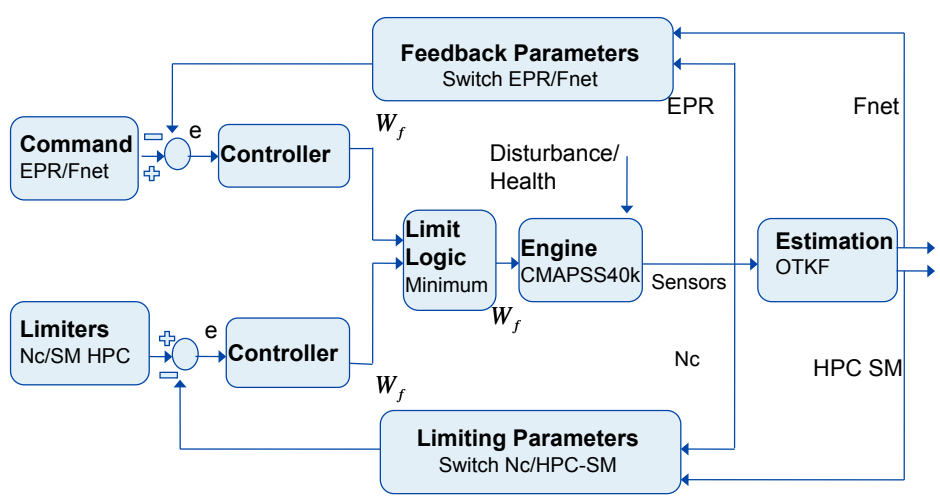

Figure 2. MBEC engine control model architecture. board model to reflect the effects of engine performance deterioration, is less than the number of unknown health parameters. In the MEBC research done to date, a subset of these health parameters equal to the number of available sensors, referred to as tuning parameters, is used to update the on-board model. Although this approach results in good model estimates of the measured variables, it does not necessarily guarantee a good estimate of the unmeasured variables such as thrust and stall margin.

Under the research being done by NASA's Aviation Safety Program, the OTKF approach has been developed to select the tuning parameters which minimize the estimation error for the unmeasured variables of interest. ${ }^{6}$ Developing a MBEC methodology around this OTKF approach provides the capability to overcome the inadequacies of past approaches and makes it practical to achieve the potential benefits of MBEC. Given the updated tuning parameters, a new control methodology can be implemented for directly controlling the thrust instead of $N_{f}$ or EPR. The following subsections will detail the engine simulation, on-board engine model, and the controller with limit logic.

\section{Engine model}

In this study, a linear point model extracted from the CMAPSS40k simulation at a cruise operating point is used to represent the engine and the MBEC on-board model. The CMAPSS40k simulation is a 40,000 lbf class turbofan engine simulation that is specifically designed for the development and testing of control algorithms. The model contains the typical suite of sensors for turbofan engine control architectures, which includes:

1. $N_{c}$, core speed

2. $N_{f}$, fan speed

3. P2, inlet pressure

4. T2, inlet temperature

5. Ps3, compressor discharge static pressure

6. T3, compressor discharge temperature

7. T48, turbine exit temperature

8. P5, turbine exit pressure

In addition, the CMAPSS40k simulation contains a fleet average profile of engine deterioration versus number of flight cycles. A $0 \%$ deterioration corresponds to a new engine, whereas a $100 \%$ deterioration implies an end of life engine. The nominal condition for this paper will be the cruise operating point of $30,000 \mathrm{ft}$ altitude, Mach 0.7 , a PLA of $60^{\circ}$, and $50 \%$ deterioration. Table 1 shows that the match between the nonlinear 
and linear simulation is highly accurate for small perturbations about the cruise condition of $2.45^{\circ}$ PLA changes, with the maximum deviation being less than 1\%. The PLA change was chosen to correspond to approximately a $10 \%$ change in steady state thrust.

Table 1. Maximum percent difference during PLA transient between the nonlinear CMAPSS40k and linear CMAPSS40k simulation for key parameters.

\begin{tabular}{|c|c|c|c|c|c|}
\hline Thrust & Ps3 & P50 & Nf & HPC SM & T48 \\
\hline $0.01 \%$ & $0.03 \%$ & $0.03 \%$ & $0.02 \%$ & $0.53 \%$ & $0.06 \%$ \\
\hline
\end{tabular}

\section{Optimal tuner Kalman Filter development}

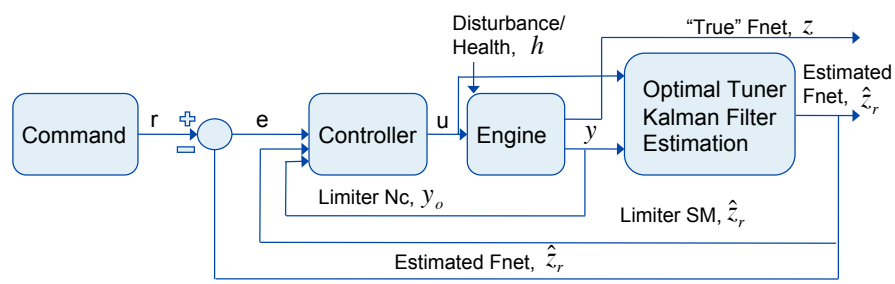

Figure 3. Simplified MBEC simulation architecture diagram to illustrate OTKF equation development.
The methodology for designing the OTKF was previously developed under NASA's Integrated Vehicle Health Management (IVHM) project. Since the IVHM project was focused on health management, previous implementations of the OTKF did not include the control parameters required for MBEC. The first step in applying the OTKF in the MBEC architecture was to account for the control command using a continuous time simulation. The change allowed for the linear Kalman Filter estimation to take into account changes in the control commands (the fuel flow) and provide an accurate estimate of thrust and stall margin.

For completeness, the main equations for the development of the OTKF are presented, ${ }^{6}$ which correspond to the MBEC block diagram shown in Fig. 3. The state vector, $x$, consists of fan and core speeds, and the control input, $u$, represents the fuel flow, VSV, and VBV. As mentioned previously though, the VSV and VBV are held at constant values during the simulation about the cruise operating point. The measured output, $y$, represents all of the sensed parameters in the system. The parameters, $z$, represent the values of thrust and stall margin. The vector, $h$, represents the health parameters of the engine. By changing the health parameters based on the percent deterioration of the engine, various stages of the engine life cycle can be simulated. To account for the deterioration the efficiency and flow capacity are modified in each of the following engine components:

1. Engine fan

2. Engine low pressure compressor

3. Engine high pressure compressor

4. Engine high pressure turbine

5. Engine low pressure turbine

For the optimal tuner estimation all of the sensors in the previous section are used with the exception of P50, as it was determined not to be required to get an accurate estimate. The total of ten health parameters and seven sensors, represents an underdetermined estimation problem thus illustrating the need for the OTKF approach. The overall engine plant model is described by a linear time-invariant state space system described in Eq. 1 to Eq. 3. To establish the Kalman Filter, uncorrelated zero-mean white noise is introduced represented by $w$ and $v$, which then establishes the covariance matrices $Q$ and $R$ respectively. The system matrices of $A, B, C, D, L, M, F, G$, and $N$ are of appropriate size to establish the system.

$$
\begin{aligned}
\dot{x} & =A x+B u+L h+w \\
y & =C x+D u+M h+v \\
z & =F x+G u+N h
\end{aligned}
$$


To account for how the health parameters induce shifts in the engine state variables, the health parameter vector can be directly concatenated with the state vector. The health parameters are modeled without dynamics since the gradual deterioration over the life of an engine is much slower than the other state variables. In an augmented form, the system is now shown in Eq. 4 to Eq. 6, where the subscript " $x h$ " denotes the combined state and health vectors.

$$
\begin{aligned}
\dot{x}_{x h}=\left[\begin{array}{c}
\dot{x} \\
\dot{h}
\end{array}\right] & =\underbrace{\left[\begin{array}{cc}
A & L \\
0 & 0
\end{array}\right]}_{A_{x h}} \underbrace{\left[\begin{array}{c}
x \\
h
\end{array}\right]}_{x_{x h}}+\underbrace{\left[\begin{array}{c}
B \\
0
\end{array}\right]}_{B_{x h}} u+\underbrace{\left[\begin{array}{c}
w \\
w_{h}
\end{array}\right]}_{w_{x h}} \\
& =A_{x h} x_{x h}+B_{x h} u+w_{x h} \\
y & =\underbrace{\left[\begin{array}{ll}
C & M
\end{array}\right]}_{C_{x h}} \underbrace{\left[\begin{array}{l}
x \\
h
\end{array}\right]}_{x_{x h}}+D u+v \\
& =C_{x h} x_{x h}+D u+v \\
z & =\underbrace{\left[\begin{array}{ll}
F & N
\end{array}\right]}_{F_{x h}} \underbrace{\left[\begin{array}{l}
x \\
h
\end{array}\right]}_{x_{x h}}+G u \\
& =F_{x h} x_{x h}+G u
\end{aligned}
$$

To account for the health parameter underdetermined estimation problem, a tuning vector is used defined by Eq. 7 .

$$
q=V^{*} h
$$

Here, $V^{*}$ is a transformation matrix that is applied to construct the tuning vector. A pseudo-inverse of the transformation matrix, $V^{* \dagger}$, is used to obtain an approximation of the health parameter vector.

$$
\hat{h}=V^{* \dagger} q
$$

More details of the optimal tuner approach can be found in Simon. ${ }^{6}$ This relationship between the health parameters and tuners leads to the following reduced order system:

$$
\begin{aligned}
\dot{x}_{x q}=\left[\begin{array}{c}
\dot{x} \\
\dot{q}
\end{array}\right] & =\underbrace{\left[\begin{array}{cc}
A & L V^{* \dagger} \\
0 & 0
\end{array}\right]}_{A_{x q}} \underbrace{\left[\begin{array}{c}
x \\
q
\end{array}\right]}_{x_{x q}}+\underbrace{\left[\begin{array}{c}
B \\
0
\end{array}\right]}_{B_{x q}} u+\underbrace{\left[\begin{array}{c}
w \\
w_{q}
\end{array}\right]}_{w_{x q}} \\
& =A_{x q} x_{x q}+B_{x q} u+w_{x q} \\
y & =\underbrace{\left[\begin{array}{ll}
C & M V^{* \dagger}
\end{array}\right]}_{C_{x q}} \underbrace{\left[\begin{array}{c}
x \\
q
\end{array}\right]}_{x_{x q}}+D u+v \\
& =C_{x q} x_{x q}+D u+v \\
z & =\underbrace{\left[\begin{array}{ll}
F & N V^{* \dagger}
\end{array}\right]}_{F_{x q}} \underbrace{\left[\begin{array}{l}
x \\
q
\end{array}\right]}_{x_{x q}}+G u \\
& =F_{x q} x_{x q}+G u
\end{aligned}
$$

The Kalman Filter is developed using a steady state approach, where the state estimation covariance and the Kalman gain do not change in time. By solving the Ricatti equation in Eq. 12 for $P$, the Kalman gain can be obtained in Eq. 13. An estimation of the reduced order state vector is then obtained by Eq. 14 . 


$$
\begin{aligned}
0 & =-P C_{x q}^{T} R^{-1} C_{x q} P+A_{x q} P+P A_{x q}^{T}+Q_{x q} \\
K & =P C_{x q}^{T} R^{-1} \\
\dot{\hat{x}}_{x q} & =A_{x q} \hat{x}_{x q}+B_{x q} u+K\left(y-C_{x q} \hat{x}_{x q}-D u\right) \\
& =\left(A_{x q}-K C_{x q}\right) \hat{x}_{x q}+K y+\left(B_{x q}-K D\right) u
\end{aligned}
$$

The controller is defined by Eq. 15 to Eq. 19

$$
\begin{aligned}
\dot{x}_{c} & =A_{c} x_{c}+B_{c e} e+B_{c o} y_{o} \\
u & =C_{c} x_{c} \\
e & =r-\hat{z}_{r} \\
\hat{z}_{r} & =\left[\begin{array}{ll}
F_{r} & N_{r} V^{* \dagger}
\end{array}\right] \hat{x}_{x q}+G_{r} u \\
y_{o} & =C_{o} x+D_{o} u+M_{o} h
\end{aligned}
$$

Combining the above controller relationships with the Kalman Filter estimate gives Eq. 20.

$$
\begin{aligned}
\dot{x}_{c} & =\left(B_{c o} C_{o}\right) x+\left(A_{c}-B_{c e} G_{r} C_{c}+B_{c o} D_{o} C_{c}\right) x_{c}-B_{c e}\left[\begin{array}{ll}
F_{r} & N_{r} V^{* \dagger}
\end{array}\right] \hat{x}_{x q}+B_{c o} M_{o} h+B_{c e} r \\
& =A_{1} x+A_{2} x_{c}+A_{3} \hat{x}_{x q}+L_{1} h+B_{c e} r
\end{aligned}
$$

Finally, combining the augmented plant with the Kalman Filter and the controller, the closed loop system can be written as:

$$
\left[\begin{array}{c}
\dot{x} \\
\dot{x}_{c} \\
\dot{\hat{x}}_{x q} \\
\dot{h}
\end{array}\right]=\left[\begin{array}{cccc}
A & B C_{c} & 0 & L \\
A_{1} & A_{2} & A_{3} & L_{1} \\
K C & B_{x q} C_{c} & \left(A_{x q}-K C_{x q}\right) & K M \\
0 & 0 & 0 & 0
\end{array}\right]\left[\begin{array}{c}
x \\
x_{c} \\
\hat{x}_{x q} \\
h
\end{array}\right]+\left[\begin{array}{c}
0 \\
B_{c e} \\
0 \\
0
\end{array}\right] r
$$

\section{MBEC architecture development}

Given the engine and on-board engine estimation elements being developed, the thrust controller and stall margin limit logic can next be developed to obtain the overall MBEC architecture. In Fig. 2 the MBEC simulation block diagram is illustrated. The thrust controller is directly closed around the estimated thrust and surge margin information is provided to the controller limit logic allowing for a reduction in the conservative margins pro-

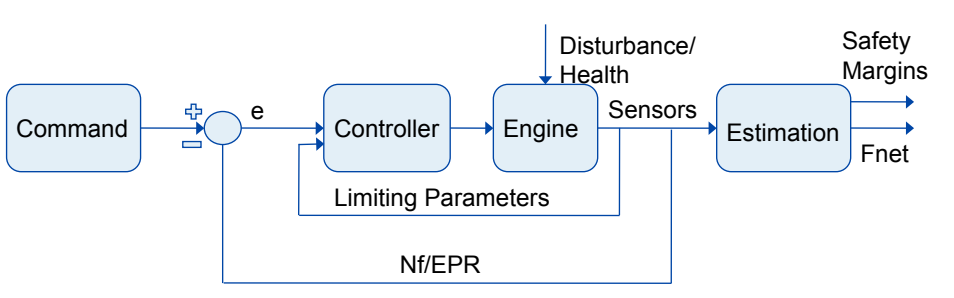

Figure 4. Engine control architecture with estimation running open loop.

viding a more efficient operation of the turbofan engine. The model accepts changes in the PLA and converts them to changes in the thrust set point. The thrust controller then uses the error signal to send the fuel flow command to the fuel valve actuator. The engine responds to the controller commands and health condition disturbances, and outputs the typical sensed parameters to the OTKF and the controller limit logic. The OTKF then provides an estimate of thrust and stall margin.

To develop the overall MBEC architecture, the linear point model of CMAPSS40k and the OTKF are first put into a MATLAB@ Simulink $($ B open loop simulation to verify the ability of the OTKF to track thrust perturbations. In Fig. 4, a general diagram of the CMAPSS40k linear model and controller is presented with the OTKF running open loop. A $2.45^{\circ}$ PLA perturbation changes the command set point for the controller. The typical EPR control architecture is used here as the feedback control. 
A standard set of sensors from the engine are used in the traditional feedback control. The OTKF also uses the standard set of engine sensors to provide estimates of non-sensed parameters such as stall margin and thrust. Here, the OTKF is simply incorporated in an open loop sense in that none of its estimated parameters are used in the control of the engine. The performance of the OTKF is illustrated in Fig. 5 and Fig. 6, where it is shown that the estimation of thrust and HPC stall margin tracks the linear model very well.
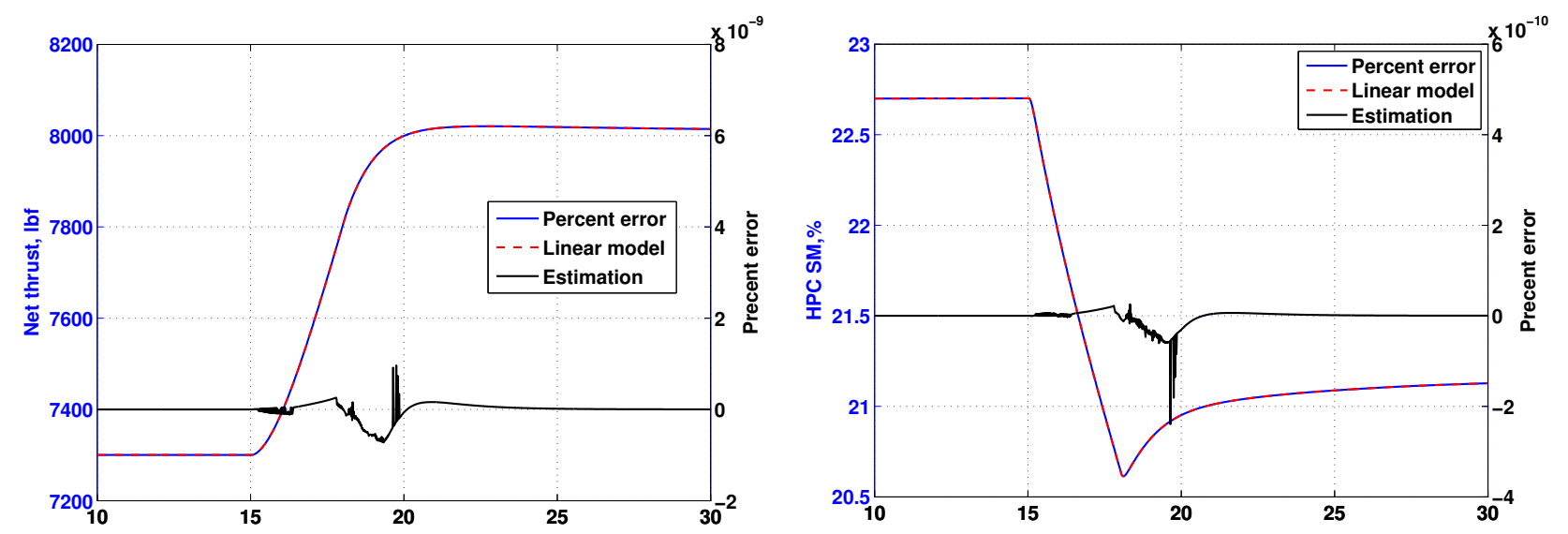

Figure 5. Verification of the optimal tuner estimation to be able to track an open loop thrust transient from the linear model at $50 \%$ deterioration.

Figure 6. Verification of the optimal tuner estimation to be able to track an open loop stall margin transient from the linear model at $\mathbf{5 0 \%}$ deterioration.

In order to develop a control algorithm based on the feedback of thrust, a linear platform model is developed representing the linear engine model operating under conventional closed-loop sensed EPR feedback control. A block diagram representation of the platform model is illustrated in Fig. 7. The engine outputs the "true" or ideal thrust, which is directly used as a feedback parameter to design a PI controller. By not having the OTKF in the platform model, this is a simpler process for developing the control algorithm. This is possible due to the highly accurate estimation as shown in Fig. 5. New controller gains for the thrust feedback control architecture are obtained by using the platform model, replacing the gains for the $N_{f}$ or EPR controller traditionally used. The same limit logic and integrator windup protection for the traditional controller are used here in developing the thrust PI controller. This will allow for the OTKF to be implemented in the closed loop MBEC simulation.

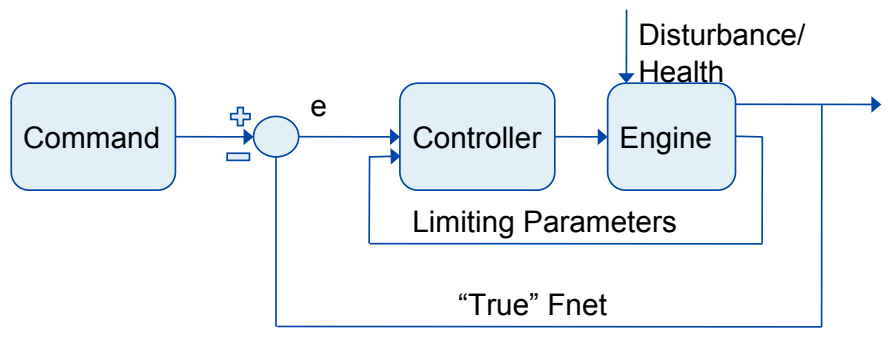

Figure 7. Ideal thrust closed loop feedback architecture.

To take advantage of the OTKF estimate of the stall margin, modifications of the traditional limit logic are required. In CMAPSS40k, HPC stall margin protection is provided by an acceleration schedule for engine core speed. This prevents the engine from surging during transient operations. Steady state surge margin is taken into account in the development of the HPC operation working line. The HPC operating line for the CMAPSS40k model was designed to provide approximately $21 \%$ nominal stall margin. The choice for the amount of safety margin is to account for all of the effects listed in Table 2. 
The use of the estimated surge margin in the controller limit logic will be conducted by replacing the traditional acceleration schedule with a stall margin limiter. The goal is to show that a new limiter can be developed to ensure that a lower stall margin threshold can be used for developing a new operating line while maintaining safe operation during transient changes. By making modifications to the operating line, the goal of obtaining efficiency gains can be realized.

\section{Results}

The purpose of these simulation results is to demonstrate the MBEC architecture using a thrust controller and stall margin limit logic through the use of a test case at the cruise operating point of $30,000 \mathrm{ft}$ altitude, Mach 0.7, and PLA of $60^{\circ}$. The results shown will first focus on the thrust controller and then illustrate the stall margin limit logic. An EPR controller with acceleration limit logic is used to provide a comparison for the MBEC architecture performance.

\section{Thrust controller results}

One of the first steps in developing the MBEC thrust controller is to obtain a reasonable command signal and bounds on the errors of the estimation compared with the error in the output thrust due to deterioration using an EPR controller. This is accomplished by running the traditional control architecture through a sweep of various engine life stages starting with a new engine all the way to an end of life engine in $25 \%$ intervals. The deviation of the engine thrust using an EPR control is shown in Fig. 8. This analysis showed that the engine does not degrade in a readily predictable fashion. It was first expected that thrust would always increase as the engine deteriorated due to core temperature increases with the loss of efficiency, however this proved not to be the case. The health deterioration algorithms for the CMAPSS40k simulation show in Fig. 8 that the maximum and minimum thrust produced correspond to the $100 \%$ and $50 \%$ engine life deterioration, respectively. To illustrate the MBEC performance over the engine life cycle, the maximum and minimum values of the output thrust using a traditional control are used to provide the error bounds that the MBEC controller must maintain a tighter control than. The command signal for the thrust controller is developed to be the average of the maximum and minimum value of the thrust response at each time step from the EPR closed loop simulation.

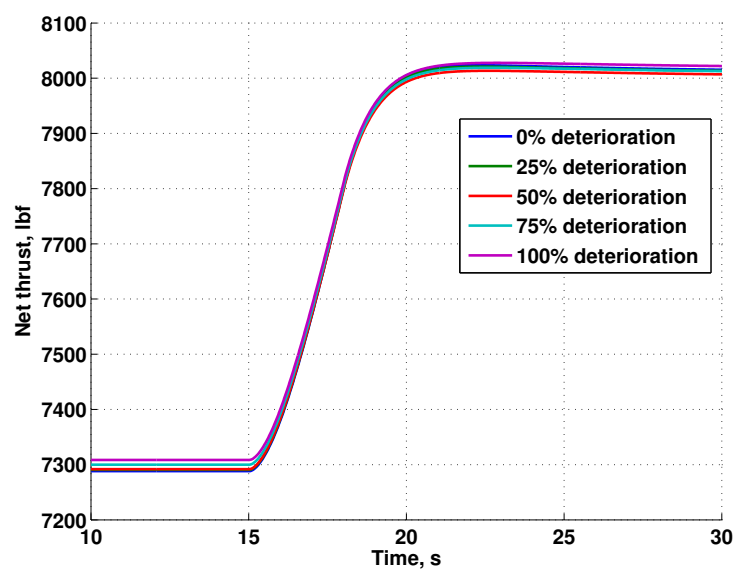

Figure 8. Engine thrust deviation over the life cycle using an EPR control algorithm and a $2.45^{\circ}$ PLA change.

The MBEC simulation is evaluated using estimated thrust for feedback control. The thrust controller performance for various deterioration levels throughout the life cycle of the engine is illustrated in Fig. 9, 
where the OTKF is designed based on the cruise linear point model for the $50 \%$ deterioration case. The upper and lower bounds shown in the figure denote the variation in net thrust that the EPR control provides over the life of the engine. The MBEC controller provides very good commanded to estimated thrust matching over the life cycle of the engine, as all of the deterioration responses lie directly on top of each other. It is clearly seen in the zoomed in portion of Fig. 9 that the thrust controller is able to maintain the estimated thrust within the EPR controller deterioration bounds. The thrust controller was deemed acceptable from this analysis and used for the remainder of the results section.
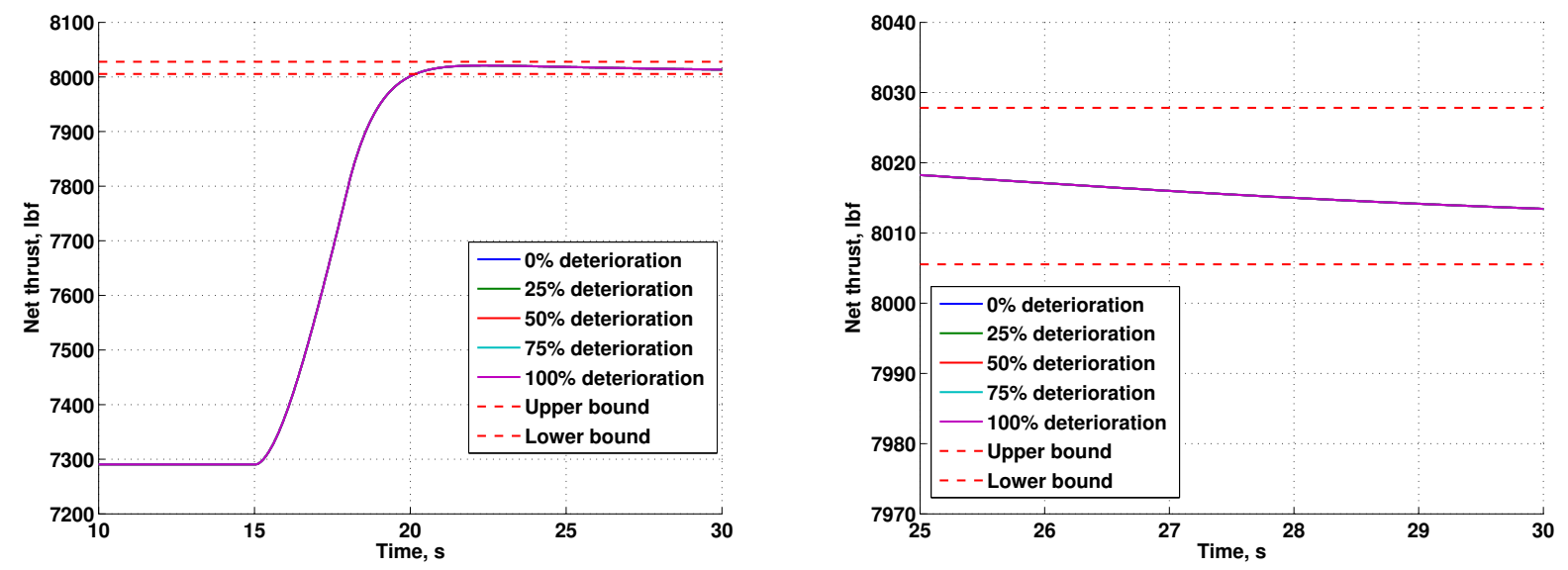

Figure 9. Estimated engine thrust controller performance over the life cycle using the MBEC thrust control algorithm with a single OTKF design and a $2.45^{\circ}$ PLA change.

The MBEC controller is based on the estimated thrust, so to determine if the MBEC control is able to provide a tighter control than the traditional EPR controller, the "true" thrust comparison is shown in Fig. 10. The same upper and lower bounds are used based on the EPR thrust deviation due to deterioration and the "true" thrust response from the MBEC estimation error is shown. The original goal was to use a single OTKF design, which has been optimized for the $50 \%$ deterioration case. As can be seen in Fig. 10, the $50 \%$ deterioration case is right in the middle of the bounds, due to the near perfect estimation that was shown previously. However, when using a single OTKF design the zoomed in portion shows that the estimation error of the OTKF over the life cycle of the engine has nearly the same deviation in "true" thrust as the EPR controller.
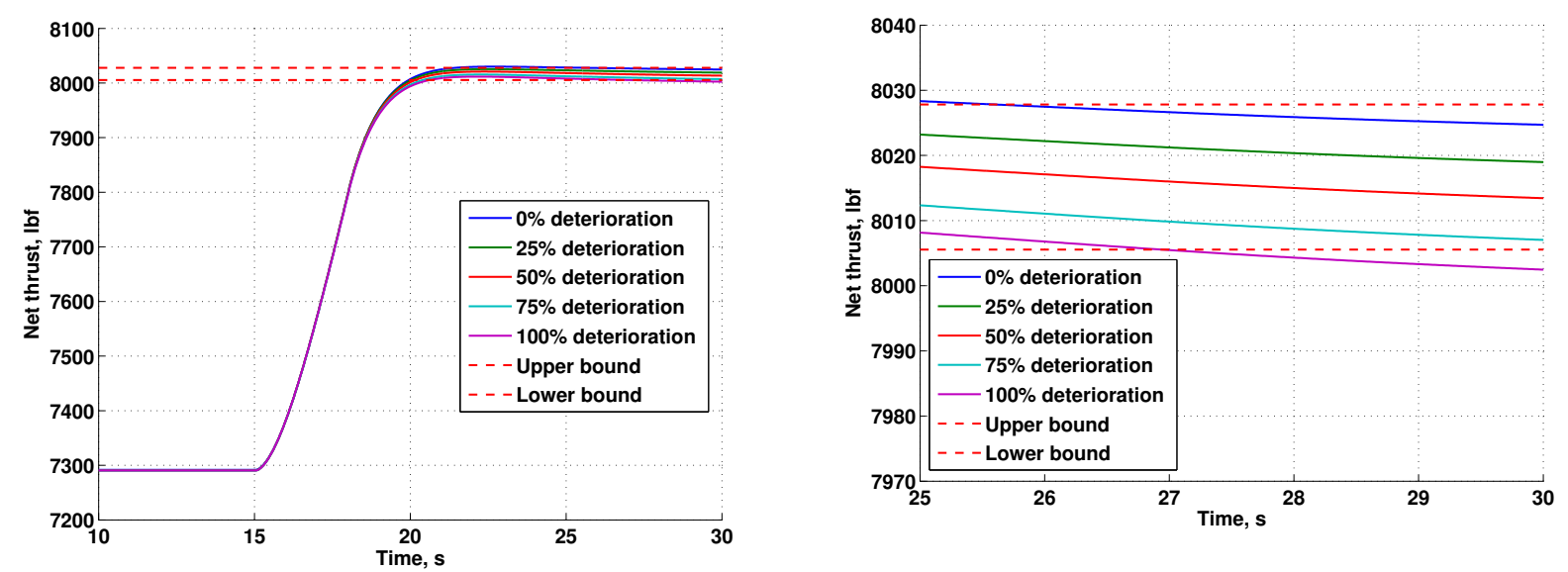

Figure 10. Engine thrust deviation over the life cycle using the MBEC thrust control algorithm with a single $V^{*}$ and a $2.45^{\circ}$ PLA change. 
Once it was determined that using a single optimal $V^{*}$ for a given operating condition would not provide a tighter control, a new optimal $V^{*}$ was calculated for each deterioration level. In Fig. 11 it is shown that a much tighter "true" thrust is obtained when the MBEC estimation is optimized for each deterioration level. This illustrates that the "true" and estimated thrust are essentially the same, as can be seen in comparing the "true" thrust shown in Fig. 11 to the controlled estimated thrust in Fig. 9. However, the assumption that the current deterioration level of the engine is known is not practical in real operation. The figure presented here is to illustrate on-going work to determine how to improve the thrust estimate, however further investigation is required for a more practical solution.
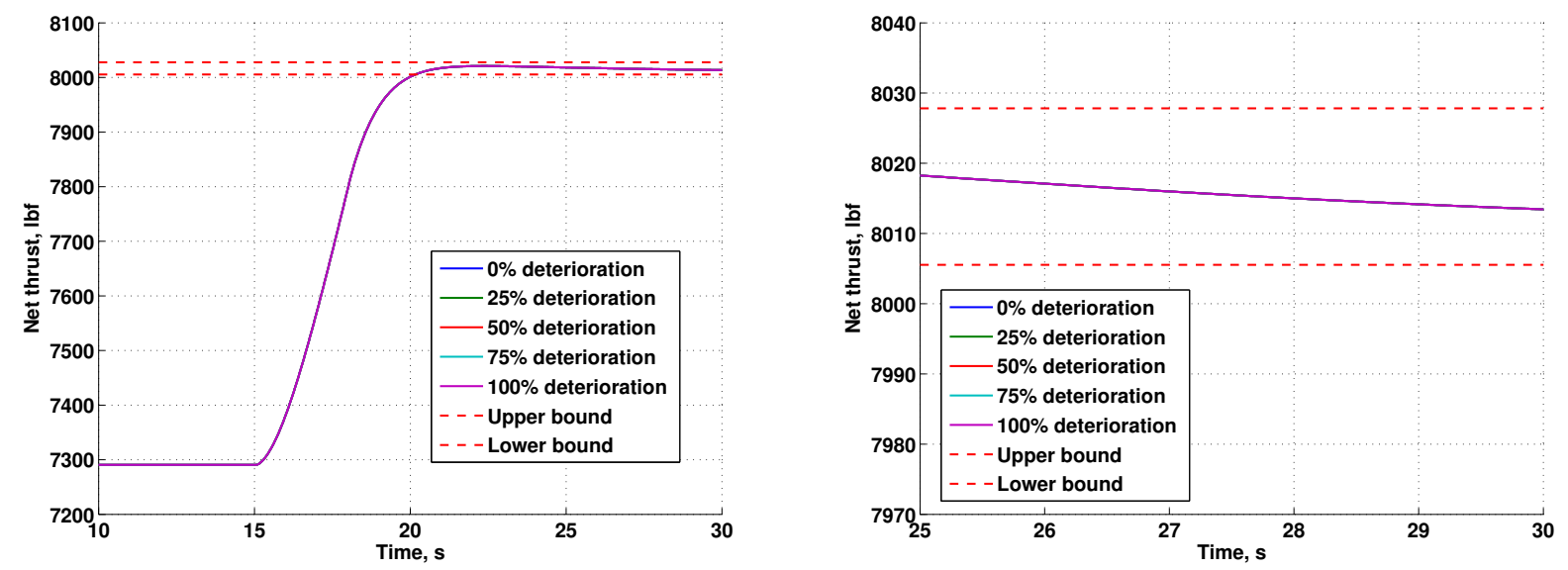

Figure 11. Engine thrust deviation over the life cycle using the MBEC thrust control algorithm with multiple OTKF designs and a $2.45^{\circ}$ PLA change.

\section{Stall margin limit logic results}

A new stall margin limiter is developed for use with the thrust controller and compared to the traditional EPR controller with an acceleration limiter. To allow for the comparison, an artificial adjustment was made to the cruise condition acceleration schedule for illustration purposes. At a given cruise condition, the size of a transient required to engage the acceleration limiter would be outside the bounds of validity for the linear model. To overcome this problem and still illustrate the impact of changing from an acceleration to a stall margin limiter, the acceleration required to trigger the limiter was reduced to engage during the previously described transient of a $2.45^{\circ}$ PLA change and a stall margin of $21 \%$.

The stall margin response due to the PLA transient using an EPR controller and acceleration schedule is shown in Fig. 12. The dashed green line illustrates the nominal response of the stall margin if there were no limiter. The red dashed line indicates the desired stall margin threshold that the acceleration schedule was artificially lowered to ensure it would engage. The blue line shows the response of the stall margin with the acceleration limiter engaged. It is clearly shown that the portion of the stall margin response that would violate the defined threshold is limited when the acceleration limit logic is engaged. The dashed black line represents the same transient without the limiter for a $100 \%$ deterioration. This illustrates the changes in stall margin due to deterioration that were shown in Table 2 and why engine safety margins for traditional control architectures need to be designed for an end of life engine.

The acceleration limiter prevents the engine from violating the threshold by limiting the fuel flow, and as a result this can have an impact on the performance of the engine. The limiter has a dramatic impact on the thrust transient as illustrated in Fig. 13. The dashed green line shows the nominal thrust response without a limiter and the blue line shows the much slower rise time due to the fuel flow being limited.

The two main components of the required stall margin shown in Table 2 are the uncertainty due to deterioration and transient response. This is the main reason for maintaining a relatively high stall margin of $21 \%$, which makes it more likely for the limiter to be engaged and impact engine performance as shown in Fig. 13. The motivation to transition to a stall margin estimated limit logic architecture for MBEC is that it has the potential to remove the uncertainty due to deterioration and transient response. 


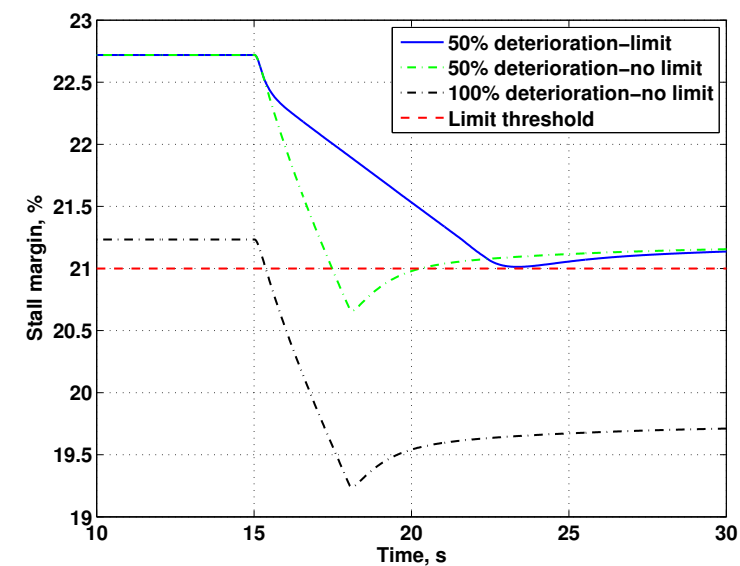

Figure 12. Engine stall margin deviation from nominal due to hitting the limiter using an EPR control and acceleration limiter.

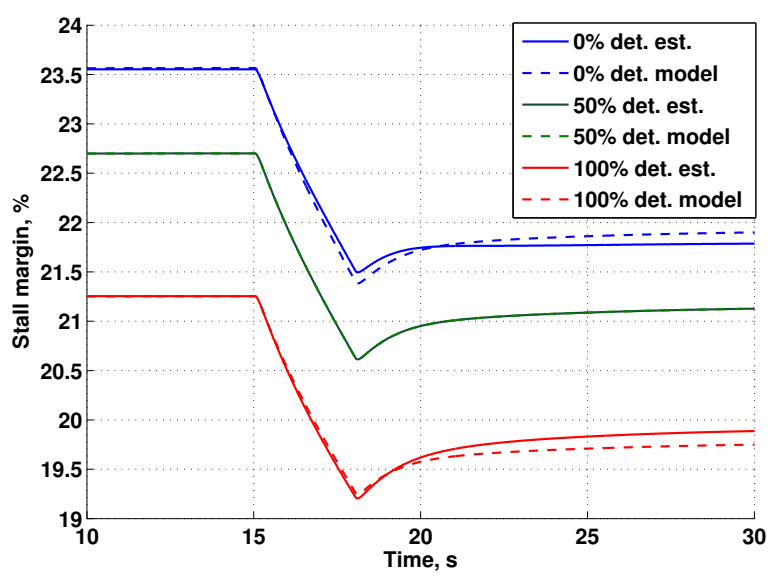

Figure 14. Engine stall margin deviation over the life cycle using a thrust control algorithm and a $2.45^{\circ}$ PLA change. stall margin threshold of $21 \%$. The blue line shows the response with the stall margin limiter. It is clearly seen that the response is able to more closely track the threshold, and then returns to the thrust based controller once the limit is no longer encountered.

The stall margin limit logic enables engine operation at the necessary level of HPC surge margin as opposed to the conventional core acceleration limit approach, which must apply more conservative logic. This allows for a tighter control, thus enabling the limit logic to have less of an impact on the engine performance if the limiter is activated. The performance of the engine based on "true" thrust response is shown in Fig. 16. The green line represents the response of the thrust without the limit, the blue line represents the MBEC thrust controller response with a stall margin limiter, and the red line is the EPR controller using the acceleration limiter. It is clearly shown that the rise time of the MBEC architecture is much shorter than the sluggish response of the EPR controller with an acceleration limiter.

The stall margin limit logic is shown to provide a significant improvement over the traditional control 
architecture, which could allow for the required safety margin to be lowered using an MBEC approach. The significance of this would be for the working line of the engine to be allowed to move to a more efficient regime of the operating envelope. This region is typically excluded due to stall margin requirements. If the engine is able to operate more efficiently by the redesign of the working line then the SFW goal of improving the thrust specific fuel consumption can be obtained.

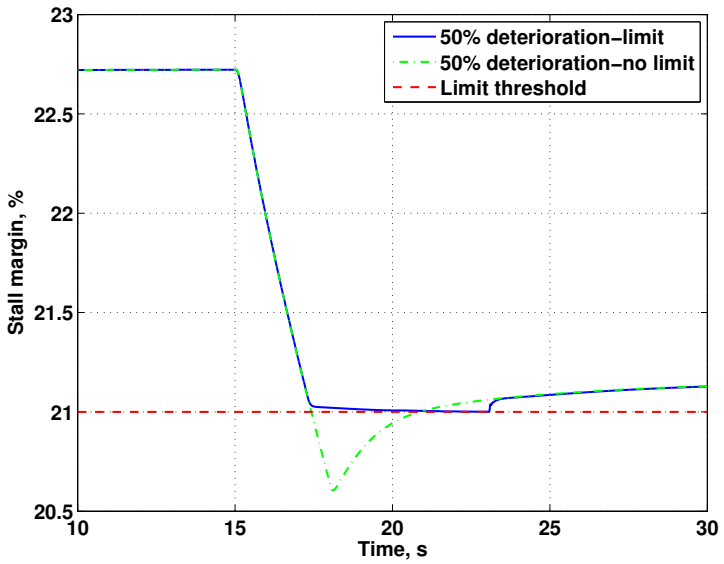

Figure 15. Engine stall margin limiter using the thrust based controller given a $2.45^{\circ}$ PLA change and 21 stall margin threshold setting.

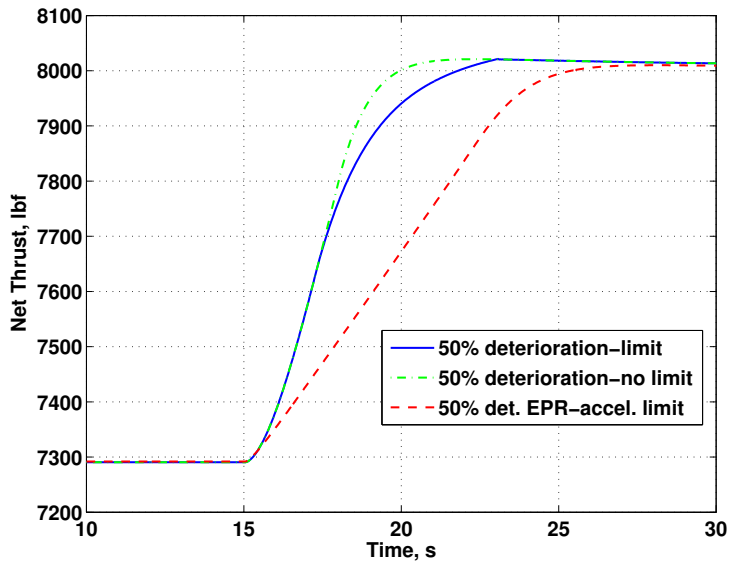

Figure 16. Engine "true" thrust deviation from nominal due to hitting the limiter using a thrust control with stall margin limiter and EPR control with acceleration limiter.

\section{Future Work}

The work presented here illustrated the potential for an MBEC architecture, however to show that this technology is viable it will be required to develop the approach on the fully nonlinear CMAPSS40k simulation. The control architecture will need to be developed for a full flight envelope and move from a simple single input - single output design to a multiple input - multiple output design to account for the VSV and VBV. Further, investigation is also required to determine the appropriate optimal design points needed to obtain the desired accuracy for the OTKF estimation.

\section{Conclusion}

The development of a linear model-based engine control (MBEC) design utilizing a cruise operating point linear model extracted from the Commercial Modular Aero-Propulsion System Simulation 40,000 (CMAPSS40k) as the turbofan engine and an optimal tuner Kalman Filter (OTKF) estimation routine as the on-board model was shown. The focus was on developing a MBEC model using an OTKF estimation of thrust and stall margin to replace traditional turbofan engine feedback control and limiting parameters. The MBEC simulation was shown to provide approximately the same control bound as is achievable using traditional control feedback for thrust over the entire life cycle of the engine using a single optimal design for the OTKF. The most significant result shown is the highly accurate estimation of the stall margin. This allowed for the development of a stall margin limit logic that improved the transient response over a traditional control architecture. In addition, the improved performance of the stall margin limit logic could allow for the reduction in safety margins, allowing for the redesign of the engine working line to a more efficient part of the operating envelope.

\section{References}

\footnotetext{
1 "NASA Fact Sheet: Energy, Efficiency and Emissions." Tech. Rep. NASA/NF-2009-08-488-HQ, NASA, 2009.

${ }^{2}$ Spang, H. A. and Brown, H., "Control of Jet Engines," Control Engineering Practice, Vol. 7, No. 9, 1999, pp. $1043-1059$.
} 
${ }^{3}$ Garg, S., Kumar, A., Mathews, H. K., Rosenfeld, T., Rybarik, P., and Viassolo, D., "More Intelligent Gas Turbine Engines," Intelligent Control and Health Monitoring, No. TR-AVT-128, chap. 3, NATO Research and Technology Organization, 2009.

${ }^{4}$ May, R., Csank, J., Litt, J. S., and Guo, T., "Commercial Modular Aero-Propulsion System Simulation 40K," Tech. Rep. NASA/TM 2010-216810, NASA, 2009.

${ }^{5}$ Csank, J., Ryan, M., Litt, J. S., and Guo, T., "Control Design for a Generic Commercial Aircraft Engine," Tech. rep., NASA/TM 2010-216811, 2010.

${ }^{6}$ Simon, D. L. and Garg, S., "Optimal Tuner Selection for Kalman Filter-Based Aircraft Engine Performance Estimation," Tech. Rep. NASA/TM 2010-216076, NASA, 2010.

${ }^{7}$ Fuller, J. W., Kumar, A., and Millar, R. C., "Adaptive Model Based Control of Aircraft Propulsion Systems: Status and Outlook for Naval Aviation Applications," ASME Turbo Expo: Power for Land, Sea and Air, No. GT2006-90241, May 8-11 2006.

${ }^{8}$ Walsh, P. P. and Fletcher, P., Gas Turbine Performance, ASME Press, Blackwell Publishing, NJ, 2nd ed., 2004. 